\title{
Multifunctional Role of TRAIL in Atherosclerosis and Cardiovascular Disease
}

\author{
Katsuhito Mori, Masanori Emoto and Masaaki Inaba \\ Department of Metabolism, Endocrinology and \\ Molecular Medicine Osaka City University \\ Graduate School of Medicine, Osaka \\ Japan
}

\section{Introduction}

Tumor necrosis factor (TNF)-related apoptosis-inducing ligand (TRAIL) belongs to the TNF ligand superfamily. As the name suggests, TRAIL was identified and cloned based on its sequence homology to the extracellular domain of TNF ligand family members (Pitti et al., 1996; Wiley et al., 1995). Human TRAIL consists of 281 amino acids. TRAIL is a type II transmembrane protein with a short intracellular amino-terminal domain and an extracellular carboxy-terminal domain (Fig. 1). TRAIL is cleaved by cysteine proteases from the cell surface to form a soluble ligand, and when released soluble TRAIL is measurable in human blood. TRAIL contains cysteine residue (Cys 230) that interacts with the zinc ion, resulting in the formation of a TRAIL homotrimer (Hymowitz et al., 1999) (Fig. 1). This trimerization is necessary for TRAIL to bind to its receptors and to exert optimal biological activity, as is described below. (Hao et al., 2004; Johnstone et al., 2008; Wang, 2008; Wu et al., 2004).

TRAIL binds to five different receptors found on a variety of cells. Of these receptors, two, TRAIL-R1 (DR4) and TRAIL-R2 (DR5), contain a cytoplasmic death domain and trigger TRAIL-induced apoptosis. Two decoy receptors lacking a functional death domain, TRAILR3 (DcR1) and TRAIL-R4 (DcR2), compete with TRAIL-R1 and TRAIL-R2 for TRAIL binding, possibly antagonizing apoptotic signaling. In addition, osteoprotegerin (OPG) is a fifth soluble decoy receptor. Briefly, the TRAIL apoptosis pathway is initiated by the binding of a TRAIL trimer to TRAIL-R1 or TRAIL-R2, which leads to receptor trimerization. This receptor conformational change recruits the adaptor protein Fas-associated death domain (FADD) through the death domains (DD) of each protein. Subsequently, FADD interacts with caspase- 8 and/or -10 through the death effector domain (DED) of each protein, resulting in the assembly of a death-inducing signaling complex (DISC). In the type I pathway, extrinsic signals proteolytically activate caspase- 8 and/or -10 followed by stimulation of effector caspase-3 and -7, the key mediators of apoptosis, triggering apoptosis. In the type II intrinsic pathway, however, apoptotic commitment requires an amplification step involving the mitochondrial pathway triggered by caspase-8-dependent cleavage of the Bid protein to its active form, t-Bid (Corallini et al., 2006; Hao et al., 2004; Testa, 2010; Wang, 2008). Since TRAIL-R3, TRAIL-R4, and OPG lack intracellular functional 
portions, it is conceivable that they do not mediate apoptosis (Fig. 2.). However, even binding of TRAIL to death receptors such as TRAIL-R1 or TRAIL-R2 can antagonize apoptosis and induce cell proliferation under certain conditions in some cells. For example, TRAIL-R1 and TRAIL-R2 recruit receptor-interacting protein (RIP) through their DD, which can activate nuclear factor $\kappa \mathrm{B}(\mathrm{NF \kappa B})$. RIP-mediated $\mathrm{NF \kappa B}$ activation appears to induce cell proliferation by TRAIL (Hao et al., 2004). TRAIL triggers recruitment of cellular FLICE-like inhibitory protein (cFLIP) to DISC through their DED in some instances. Some cFLIPs appear to prevent TRAIL-induced apoptosis (Hao et al., 2004; Wang, 2008). TRAIL stimulation also recruits phosphoprotein enriched in diabetes (PED) to the DISC. This process inhibits downstream caspase activation in some cases (Hao et al., 2004) (Fig. 2.). Therefore, two contradictory steps should be considered when determining the biological effects of TRAIL besides simple apoptotic signaling. First, TRAIL has death receptors (TRAIL-R1 and TRAIL-R2) and antagonized decoy receptors (TRAIL-R3, TRAIL-R4, and OPG) at the cell surface level. Second, these death receptors can counteract apoptotic signaling at the intracellular level in certain contexts. Thus, TRAIL signaling is very complicated.

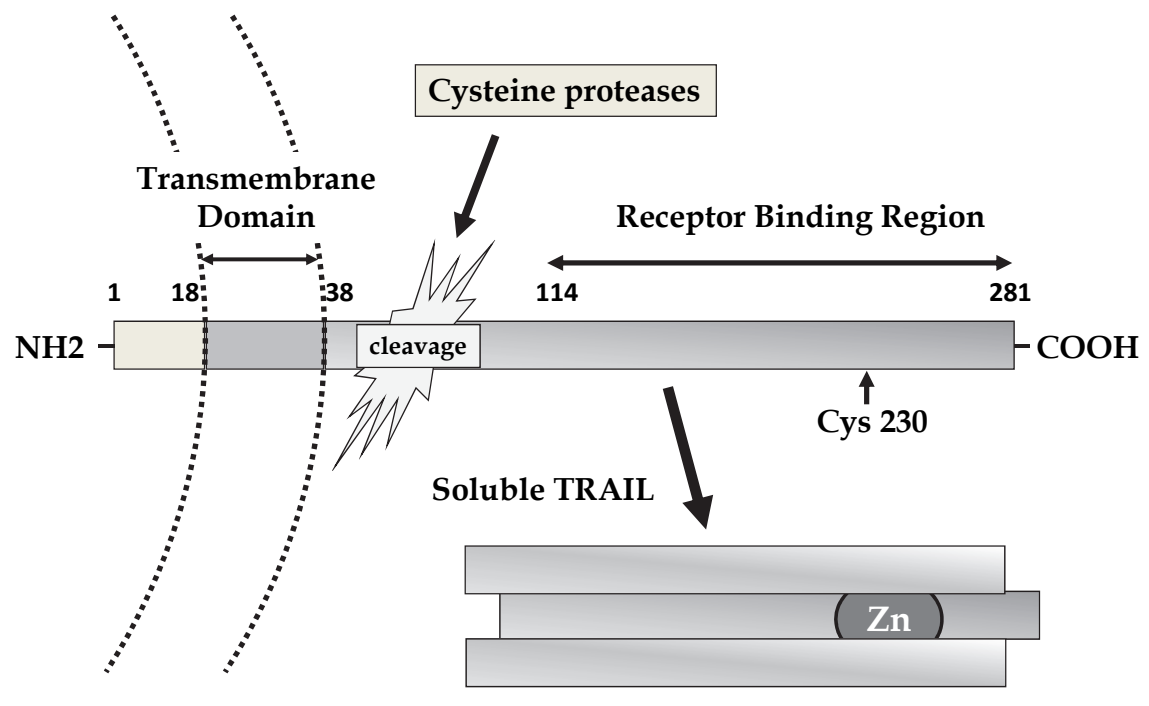

Formation of homotrimer

TRAIL consists of 281 amino acids with a transmembrane domain that separates the short intracellular and extracellular domains. The extracellular domain of TRAIL is cleaved and released into the bloodstream. TRAIL forms a homotrimer through interactions between zinc ions and each cysteine residue.

Fig. 1. The Structure of TRAIL.

TRAIL has attracted clinical attention as a promising agent for the treatment of cancer since it can induce apoptosis in various tumor cells without having any toxic effects on normal cells (Finnberg and El-Deiry, 2008; Wang, 2008; Wu et al., 2004). Apart from high expectations in the field of cancer, TRAIL also exhibits diverse biological effects on the immune system, hematopoiesis, and metabolic disorders, including diabetes (Afford and 
Adams, 2005; Benito-Martin et al., 2009; Corallini et al., 2006; Testa, 2010; Vaccarezza et al., 2007). Recently, the involvement of TRAIL in atherosclerosis and cardiovascular diseases has been at the forefront of research efforts (Corallini et al., 2008; Kavurma and Bennett, 2008; Martin-Ventura et al., 2007). In this review, we focus on the role of TRAIL in the cardiovascular system.

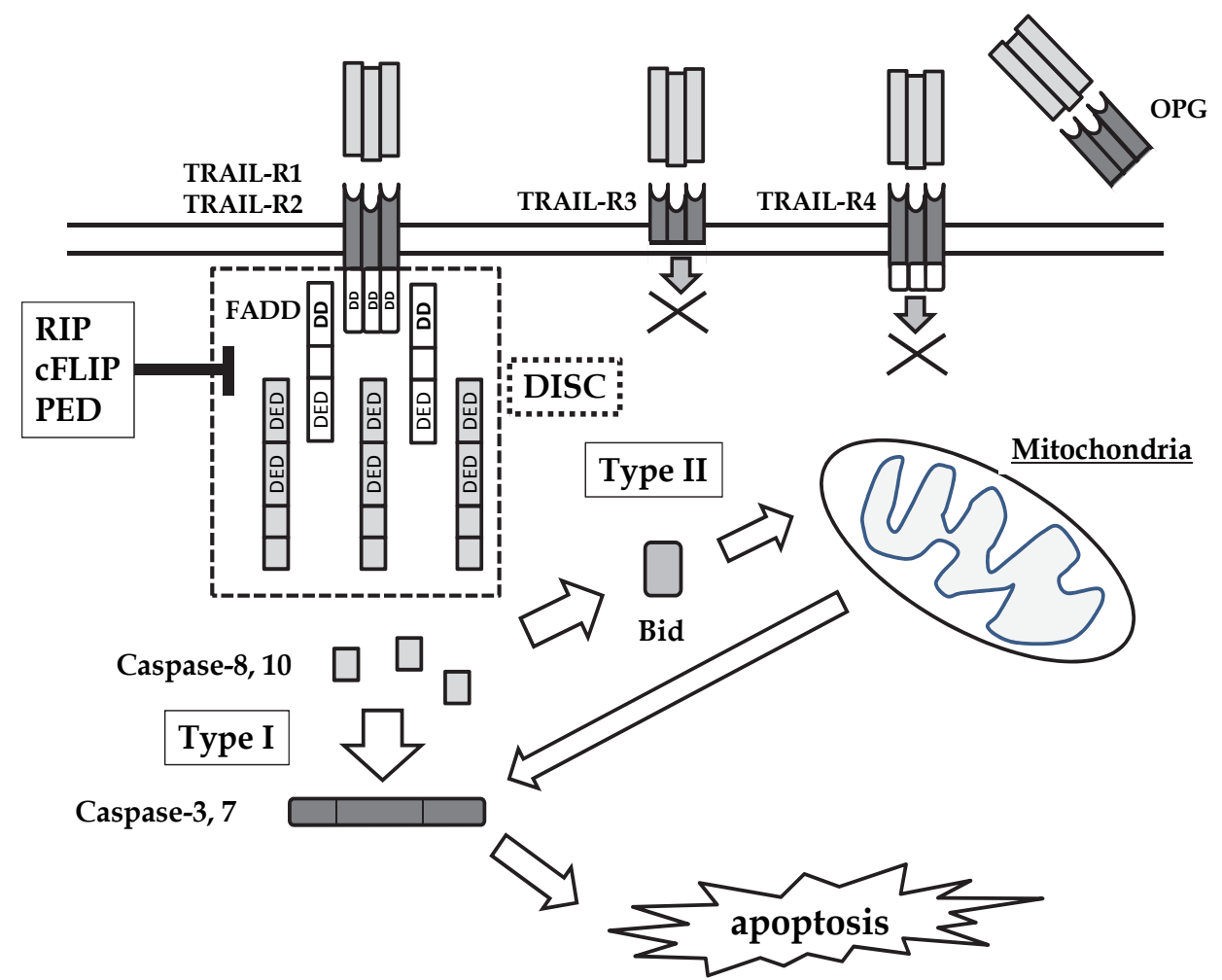

Among the five known TRAIL receptors, TRAIL-R1 and -R2 can induce intracellular apoptotic signaling. Upon binding of TRAIL to TRAIL-R1 or -R2, a death-inducing signaling complex (DISC) is formed through the recruitment of Fas-associated death domain (FADD), and subsequently results in the activation of caspase-8/10. In the type I intrinsic pathway, this activation is sufficient to conduct apoptosis through downstream effector caspases. On the other hand, activated Bid by caspase-8 amplifies apoptotic signaling through the mitochondria, which is a necessary step to induce effective downstream signaling in the type II extrinsic pathway. In addition to this activation process, it appears that at least a few other factors, such as receptor-interacting protein (RIP), cellular FLICE-like inhibitory protein (cFLIP), and phosphoprotein enriched in diabetes (PED), are antagonistic to TRAIL-induced apoptosis.

Fig. 2. TRAIL receptors and downstream apoptotic signaling.

\section{TRAIL and the cardiovascular system}

TRAIL and its receptors are known to be expressed in the cardiovascular system. Therefore, it is easily hypothesized that TRAIL systems may be involved in cardiovascular homeostasis and disorders. To begin with, we will summarize several in vitro findings that demonstrate 
the direct effects of TRAIL on each cellular component in the arterial wall. We will then discuss how TRAIL can act on the cardiovascular system based on evidence from animal models. Subsequently, we try to make causal inferences relating to TRAIL's role in cardiovascular diseases from cross-sectional and longitudinal clinical studies in humans. Finally, we report our recent clinical findings and debate whether TRAIL could be a common biomarker that reflects early to advanced stage atherosclerotic changes.

\subsection{Effects of TRAIL on cellular components in the arterial wall (in vitro findings)}

Endothelial cells (ECs) and vascular smooth muscle cells (VSMCs) are the principal cellular components of the normal arterial wall. Immune-inflammatory infiltrates such as activated $\mathrm{T}$ lymphocytes and macrophages play crucial roles in the onset and development of atherosclerosis (Libby, 2002; Weyand et al., 2008). This process is triggered and exacerbated by complicated interactions between modified lipoproteins and various cellular components. Since TRAIL and its receptors are expressed in both physiological and pathological arterial walls (Corallini et al., 2008; Kavurma and Bennett, 2008; MartinVentura et al., 2007; Schoppet et al., 2004), it is hypothesized that TRAIL signaling could regulate vascular wall homeostasis and morbid atherosclerosis. TRAIL is expressed in each cellular component, both in resident cells (ECs, VSMCs) and in infiltrating cells ( $T$ lymphocytes and macrophages) (Corallini et al., 2008; Kavurma and Bennett, 2008). To determine the role of TRAIL in atherosclerosis, we must first determine the effects of TRAIL on each cellular component based on results from in vitro studies.

ECs are located on the intima, which separates the blood stream from the vascular wall. In addition, these cells have a critical function not only as a simple barrier but also as a dynamic organ. Thus, ECs can regulate vascular tone, inflammation, thrombosis, and vascular remodeling. Balanced homeostasis in ECs protects against atherosclerosis while disruption of endothelial function results in the initiation and development of atherosclerosis (Sandoo et al., 2010). Human ECs, both human umbilical vein ECs (HUVECs) and human dermal microvascular ECs (HDMECs), express death receptors, TRAIL-R1 and TRAIL-R2. As expected, TRAIL could induce apoptosis in ECs, although this phenomenon was not observed in every cell (Li et al., 2003). This study also found that apoptosis in ECs could be reduced by treatment with caspase inhibitors and by transfection of dominant-negative FADD, suggesting the involvement of a FADD-caspase 8 pathway in this apoptotic process ( $\mathrm{Li}$ et al., 2003). Subsequently, Secchiero et al. showed that both HUVECs and primary human aortic ECs express death TRAIL-R1, -R2 and decoy TRAIL-R3, -R4. As predicted, the removal of serum and endothelial cell growth factor (tropic withdrawal) from the cell culture system led to apoptosis. Surprisingly, TRAIL protected HUVECs from tropic withdrawal-induced apoptosis. Activation of phosphatidylinositol 3kinase (PI3K)/Akt pathway by TRAIL appears to be involved in this anti-apoptotic action. In addition to this unexpected anti-apoptotic property, TRAIL stimulated proliferation of ECs in an ERK 1/2 dependent manner in this cell system (Secchiero et al., 2003). It has also been reported that TRAIL alone can increase HUVEC cell proliferation (Alladina et al., 2005). As reported above (Secchiero et al., 2003), inhibition of the PI3K/Akt pathway sensitizes EC cells to TRAIL-induced apoptosis (Alladina et al., 2005). More detailed investigations have found that inhibition of PI3K/Akt leads to activation of both extrinsic and intrinsic apoptotic pathways and appears to sensitize HUVECs to TRAIL-induced apoptosis by concurrent down-regulation of anti-apoptotic cFLIPs and Bcl-2 (Alladina et al., 
2005). Taken together, these results suggest that the effects of TRAIL on ECs appear to depend on cell culture conditions. Aside from cell apoptosis/proliferation, ECs have a protective role against atherosclerosis by producing nitric oxide (NO), one of the most important vasoactive factors. NO, a potent vasodilator, has antithrombotic and antiinflammatory activity (Sandoo et al., 2010). Interestingly, TRAIL stimulates NO production through activation of endothelial NO synthase (eNOS) in HUVECs (Zauli et al., 2003). In particular, eNOS trafficking appears to be involved in TRAIL-induced eNOS activation. TRAIL can translocate eNOS from the cell membrane to the cytoplasm in HUVECs (Di Pietro et al., 2006). However, microtubule disruption with nocodazole inhibits the eNOS activity induced by TRAIL, suggesting that translocation of eNOS through cytoskeletal reorganization may be necessary for TRAIL-induced NO production (Di Pietro et al., 2006). NO synthesis with cytoskeletal alteration by TRAIL is possibly linked to the migration of HUVECs stimulated by TRAIL treatment (Zauli et al., 2003).

In terms of atherosclerosis, attachment of blood leukocytes to ECs is recognized as the first step in the initiation of this phenomenon (Libby, 2002). It is probable that TRAIL regulates this process as well. An adhesion assay using co-cultured HUVECs and HL-60 leukocytes showed that TRAIL increased the binding of leukocytes to ECs (Li et al., 2003). A later report reproduced the pro-adhesive activity of TRAIL, although its effect was much less than that of pro-inflammatory cytokines such as TNF $\alpha$ and interleukin-1 $\beta$ (Secchiero et al., 2005). When TRAIL and pro-inflammatory cytokines were evaluated at the same time, TRAIL pretreatment showed reverse inhibition of TNF $\alpha$ - and IL 1 $\beta$ - induced HL-60 leukocyte adhesion to HUVECs. This inhibitory effect of TRAIL on cell attachment was mediated by down-regulation of the inflammatory chemokines CCL8 and CXCL10, which were stimulated by TNF $\alpha$ (Secchiero et al., 2005). In particular, TRAIL-R1 and TRAIL-R2 contributed to the TRAIL-induced down-regulation of chemokine release (Secchiero et al., 2005). Again, TRAIL has multiple context-dependent effects. At any rate, TRAIL may play an important role in EC function, both physiologically and pathologically.

Another physiological cellular component is the VSMCs, which are mainly localized in the media and constitute the vascular structure. Once VSMCs are transformed, however, they proliferate and migrate, contributing to the development of atherosclerosis (Ross, 1993). In advanced atheromatous plaques, apoptosis of VSMCs in the fibrous cap may be involved in plaque rupture. The former transformation exacerbates atherosclerosis (Libby, 2002), whereas the latter leads to poorer outcome (Clarke et al., 2006). TRAIL participates in the apoptosis and proliferation of VSMCs as well as ECs. For example, plaque-derived CD4 T cells expressing TRAIL induce apoptosis of VSMCs, probably through the interaction of TRAIL with TRAIL-R2 (Sato et al., 2006). On the other hand, TRAIL has been reported to act on human and rat VSMCs as an anti-apoptotic factor through TRAIL-R1 and -R2 (Secchiero et al., 2004). TRAIL promotes proliferation and migration of VSMCs through activation of ERK (Secchiero et al., 2004). Furthermore, Kavurma et al. reported that TRAIL stimulated proliferation of human VSMCs, although it induced apoptosis at high concentrations (Kavurma et al., 2008). Interestingly, they showed the importance of insulin-like growth factor-1 (IGF-1), one of the most potent growth factors, in TRAIL-induced proliferation of VSMCs. Thus, TRAIL up-regulates expression of the IGF-1 receptor in an NFKB-dependent manner (Kavurma et al., 2008). It is thus too soon to decide whether TRAIL causes apoptosis or proliferation in VSMCs, even in vitro.

In addition to the seemingly contradictory findings in ECs and VSMCs, a few reports have suggested that TRAIL has a pro-apoptotic effect on inflammatory lymphocytes (Janssen et 
al., 2005) and macrophages (Kaplan et al., 2000). In either case, TRAIL appears to have multiple functions in both physiological and pathological cells under various conditions. As described previously, the existence of five different types of TRAIL receptors and the crosstalk among multiple post-receptor signaling pathways may explain these diverse effects. On the basis of in vitro findings, it is very difficult to speculate the impact of TRAIL on atherosclerotic lesions in vivo, although the in vitro findings clearly indicate the involvement of the TRAIL/TRAIL receptor system in atherogenesis. In the next section, we try summarizing and discussing whether TRAIL protects against or exacerbates atherosclerosis in ways aside from its function as a mere pro-apoptotic factor.

\subsection{Role of TRAIL in atherosclerosis and vascular injury (in vivo findings)}

With regard to the role of TRAIL in the vascular wall, Secchiero et al. first demonstrated compelling in vivo findings using diabetic apolipoprotein $\mathrm{E}$ (apoE)-null mice that mimic the atherosclerotic lesions observed in humans (Secchiero et al., 2006). That is, intraperitoneal administration of recombinant human TRAIL into these mice resulted in a transient high concentration of TRAIL and subsequent protection against the development of atherosclerosis (Secchiero et al., 2006). Secchiero et al. carefully investigated the effects of TRAIL on in vivo atherosclerotic lesions using an adeno-associated virus containing TRAIL to ensure a low but sustained expression of TRAIL, similar to physiological conditions. TRAIL again attenuated the development of atherosclerotic plaques, even under these conditions (Secchiero et al., 2006). One of the mechanisms responsible for this effect may be selective apoptosis of infiltrating macrophages in plaque lesions. At the same time, increased VSMC levels were also observed in the fibrous caps of the atherosclerotic lesions. This increase may contribute to stabilization of the atherosclerotic plaques (Secchiero et al., 2006). Therefore, TRAIL appears to act as a protective factor against atherosclerosis in vivo. Subsequently, Kavurma's group showed the direct effects of TRAIL on VSMCs in vivo. They have already reported that TRAIL stimulates VSMC proliferation in vitro (Kavurma et al., 2008). To prove this using an in vivo model, they used a cuff-induced vascular injury method in TRAIL-null mice. In wild-type mice, this procedure can induce VSMC proliferation and intimal thickening in response to vascular injury. However, TRAIL-null mice were protected from neointimal formation and displayed reduced VSMC proliferation, suggesting a significant role for TRAIL in VSMC proliferation in vivo (Chan et al., 2010). Although this report showed the direct effects of TRAIL on VSMCs, the lack of atherogenic factors involved in the formation of vascular lesions should be studied further. Very recently, it has been reported that TRAIL attenuates the development of atherosclerosis using TRAIL (TRAIL -/-)/apoE (apoE -/-) double-knockout mice (Watt et al., 2011). TRAIL -/- apoE $-/-$ mice had significantly larger atheromatous lesions compared with apoE -/- control mice at 8 weeks. The larger lesions in TRAIL $-/-$ apoE $-/-$ mice appeared to be due to an increase in the number of lesional VSMCs, suggesting the anti-atherogenic action of TRAIL. Intriguingly, the difference in atheromatous lesion size among these mice became smaller at 12 weeks. In contrast to Secchiero's findings, the lack of TRAIL had no effect on the macrophage content in the atheromatous lesions. There are still unsolved and controversial issues with regard to the precise mechanisms by which TRAIL acts on the arterial wall. However, TRAIL at least appears to protect against atherogenic lesions as a whole in vivo.

\subsection{TRAIL and cardiovascular disease and prognosis (clinical findings)}

Clinical studies of the relationship between TRAIL and cardiovascular diseases are also of interest. Recently, a significant relationship has been reported by a number of studies. The 
first study demonstrated that serum TRAIL levels were significantly lower in patients with acute coronary syndrome (ACS) compared with those with stable angina and normal coronary arteries (Michowitz et al., 2005). Subsequently, Schoppet et al. found a tendency for serum TRAIL levels to be lower in patients with coronary artery disease (CAD) compared with subjects without CAD, although this difference was not significant. To further investigate this relationship, we examined serum TRAIL levels in 285 subjects who underwent coronary angiography for suspected CAD. Interestingly, we found that serum TRAIL levels were inversely associated with the severity of CAD (Mori et al., 2010). In particular, TRAIL levels in patients with severe three-vessel disease (VD) were significantly lower than in those without CAD (Mori et al., 2010). Moreover, TRAIL was an independent and negative contributor for the presence of CAD (Mori et al., 2010). Taken together, these results suggest that lower TRAIL levels may reflect the advancement of CAD.

In addition to cross-sectional findings, recent studies have also suggested that TRAIL can be a protective predictor against cardiovascular prognosis. As previously reported (Michowitz et al., 2005), Secchiero et al. showed that serum TRAIL levels were significantly lower in patients with acute myocardial infarction (AMI) at baseline (within 24 hours from admission) compared with healthy subjects (Secchiero et al., 2009). Interestingly, serum TRAIL levels at baseline were significantly lower in patients with in-hospital adverse events compared with those who did not experience these events (Secchiero et al., 2009). Subsequently, they observed that serum TRAIL levels in AMI patients gradually recovered at discharge. Furthermore, low TRAIL levels at discharge were associated with an increased incidence of cardiac death and heart failure in the 12-month follow-up (Secchiero et al., 2009). The prognostic value of TRAIL was also examined in 351 patients with advanced heart failure (HF). Again, low serum TRAIL levels were related to a worse prognosis. The risk of mortality dropped by $70 \%$ in the highest quartile of TRAIL levels, suggesting that TRAIL is a strong inverse predictor of mortality in patients with advanced HF (Niessner et al., 2009). In addition, the role of TRAIL as a more general predictor of mortality and not limited to patients with AMI or advanced HF was investigated by a large prospective population-based study of older people (Volpato et al., 2011). Baseline TRAIL levels were inversely related to all-cause mortality over a period of six years (Volpato et al., 2011). As expected, more detailed analyses revealed that the prognostic effect of TRAIL levels was strong and highly significant in subjects with prevalent cardiovascular diseases (Volpato et al., 2011). These findings expand on the predictive ability of TRAIL at a population level with a longer follow-up period. Moreover, Secchiero et al. focused not only on TRAIL but also on OPG. As was described earlier, OPG is a soluble neutralizing receptor for TRAIL. In contrast to TRAIL, it is known that serum OPG levels are positively associated with the presence and severity of CAD and are inversely correlated with prognosis (Abedin et al., 2007; Jono et al., 2002; Kiechl et al., 2004; Omland et al., 2008; Rhee et al., 2005; Schoppet et al., 2003). In this context, both TRAIL and OPG were evaluated at the same time. The researchers found that the OPG/TRAIL ratio was significantly higher in patients with acute AMI who developed HF during the follow-up period (Secchiero et al., 2010). The increase in OPG, which is a decoy TRAIL receptor, may act against TRAIL and thus negate its protective effects on the cardiovascular system. Thus, an unbalanced OPG/TRAIL ratio may be a more accurate predictor of prognosis after AMI.

\subsection{Modulating factors in TRAIL-associated biological and clinical effects}

Several reports suggest that expression and/or serum levels of TRAIL are modulated by certain factors. As described above, serum TRAIL levels drop after AMI. Since proteolytic 
enzymes including matrix metalloproteinases (MMPs) are released following AMI, researchers have investigated whether MMPs could cleave TRAIL and thus decrease TRAIL levels, inducing AMI. In addition to MMPs, Secchiero et al. have simultaneously measured tissue inhibitors of MMPs (TIMPs) that antagonize MMPs (Secchiero et al., 2010). Among the examined MMPs and TIMPs, the circulating MMP2/TIMP2 ratio showed a significant inverse correlation with serum TRAIL levels in AMI patients (Secchiero et al., 2010). Indeed, MMP2 cleaved TRAIL and abrogated its biological activity in vitro (Secchiero et al., 2010). Therefore, an elevated MMP2/TIMP2 ratio following AMI may cause degradation of TRAIL, resulting in a poorer outcome. With regards to TRAIL expression, insulin has been reported to down-regulate TRAIL expression in VSMCs both in vitro and in vivo (Corallini et al., 2007). Since VSMCs are also known to release bioactive NO in response to TRAIL, it is hypothesized that chronic insulin exposure in VSMCs may induce vascular dysfunction through TRAIL suppression in diabetic patients with hyperinsulinemia or those undergoing insulin treatment. Moreover, it has been reported that activated protein C (APC), an antithrombotic and anti-inflammatory serine protease, inhibits TRAIL expression in HUVECs (O'Brien et al., 2007). In that study, intracellular signaling was thoroughly examined (O'Brien et al., 2007). However, there has been some criticism of APC-mediated decrease of TRAIL levels. It is currently unknown whether this reflects a decrease in cellsurface TRAIL expression or of TRAIL release from its intracellular pool (Secchiero and Zauli, 2008).

Statins also appear to down-regulate TRAIL expression in cytotoxic CD4 T cells in patients with ACS, resulting in protection against destabilization of plaques (Sato et al., 2010). That is, CD4 T cells are enriched in the blood of ACS patients and induce strong apoptosis of ECs, probably through TRAIL-R2 (DR5) (Sato et al., 2010). This EC apoptosis may be involved in the erosive progression of vulnerable plaques. Interestingly, statins directly block CD4 T cell-mediated EC apoptosis (Sato et al., 2010). Therefore, this protection against endothelial injury shown by statins may explain one of the pleiotropic effects of statins in cardiovascular events. On the other hand, it has been reported that statins augment TRAIL-induced apoptosis in tumor cells but not in normal cells (Jin et al., 2002), although this is not the case in vascular cells. In this regard, pioglitazone, an anti-diabetic agent, also enhances TRAILinduced apoptosis in tumor cells (Goke et al., 2000). In clinical studies, use of various medications against diabetes, hypertension, dyslipidemia, and so on is often unavoidable. To interpret the association between TRAIL and cardiovascular lesions, it is important to pay attention to the types of interventions used.

\subsection{TRAIL as a possible biomarker in early atherosclerotic lesions}

As noted above, lower TRAIL levels appear to be correlated with more severe cardiovascular lesions and poorer prognosis. Thus, the next question is whether TRAIL levels also inversely reflect early atherosclerotic lesions. We therefore examined the association between TRAIL and intima-media thickness (IMT), which is a surrogate marker for atherosclerotic changes, using ultrasonography in 416 diabetic patients. We found no significant association between serum TRAIL levels and IMT (Kawano et al., 2011). Interestingly, when we focused on subjects with macrovascular diseases such as CAD, cerebrovascular diseases, and arteriosclerosis obliterans, there was a significant and reproducible association between TRAIL levels and carotid IMT. These findings suggest that TRAIL may not be a good candidate biomarker for early atherosclerotic lesions. However, TRAIL still appears to be a good biomarker of advanced atheroscletoic lesions. 
In contrast to IMT, which indicates morphological wall thickening, endothelial dysfunction is recognized as reflecting earlier functional vascular damage. Flow-mediated dilatation (FMD) is a representative method of non-invasive evaluation of endothelial function. This ultrasound-based method quantifies vasodilatation in response to NO production from endothelial cells induced by shear stress (Corretti et al., 2002; Patel and Celermajer, 2006; Ter Avest et al., 2007). Since in vitro findings have suggested various biological effects of TRAIL on endothelial cells (as described previously), investigation of the association between TRAIL and endothelial function is of interest. In this context, we tried measuring FMD and TRAIL levels in 109 subjects (57 men and 52 women, aged $48.4 \pm 16.6$ years). In the trial, we focused on the following two points: First, we targeted healthy subjects to avoid various confounding biases such as metabolic disorders with concomitant drug intervention. Second, we made sure of the accuracy of FMD. It is often noted that the value of FMD is highly dependent on the technique used by various institutions (Ter Avest et al., 2007). Very recently, semi-automated equipment (Unex Co. Ltd., Nagoya, Japan) has become available for evaluating FMD that gives good reproducibility (Tomiyama et al., 2008; Tomiyama and Yamashina, 2010). We used this new equipment in this study. The serum TRAIL level was measured by an enzyme-linked immunosorbent assay kit (R\&D systems, Minneapolis, USA). The mean serum TRAIL level was $75.2 \pm 20.7 \mathrm{pg} / \mathrm{ml}$, with a range of 32.4-147.4 $\mathrm{pg} / \mathrm{ml}$. The TRAIL level was not significantly correlated with FMD $(\rho=-0.128, \mathrm{p}=0.184)$ (Fig. 3) (unpublished data). Again, these findings suggest that TRAIL may not be a good candidate as a biomarker of early atherosclerotic lesions.

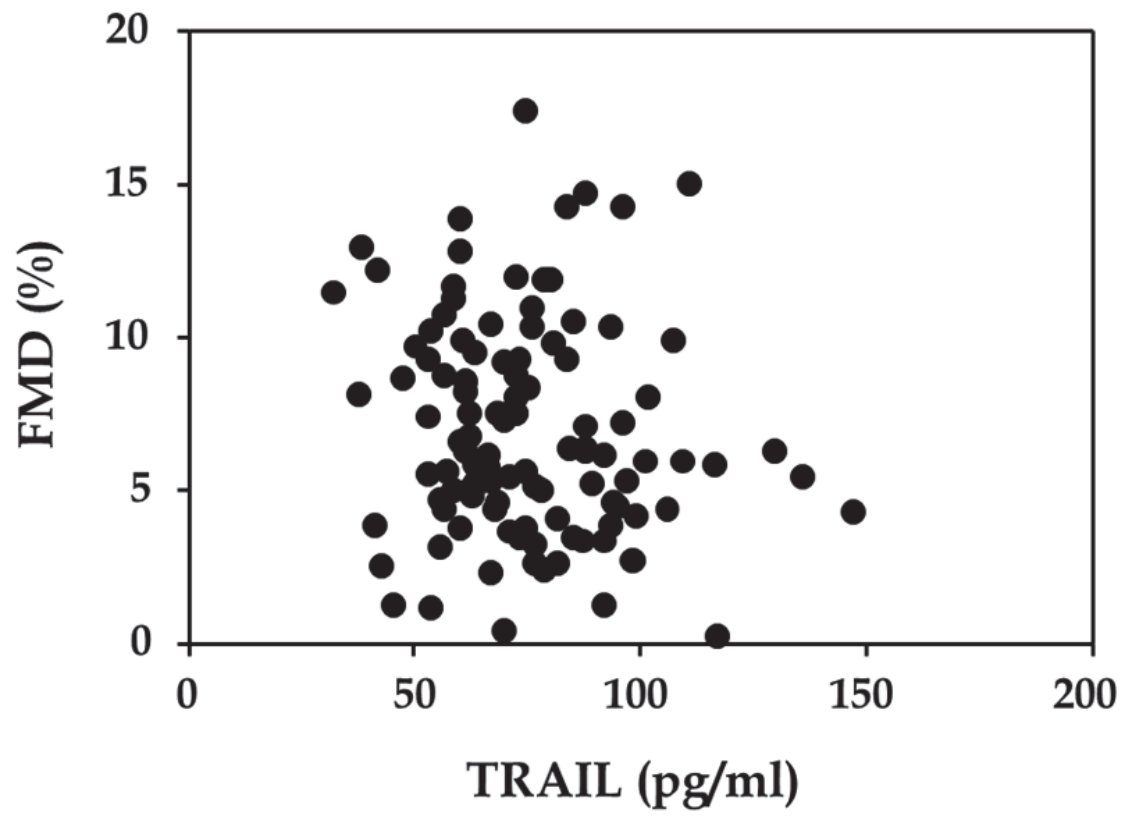

Fig. 3. The association of TRAIL with endothelial function in 109 healthy subjects.

Flow-mediated dilatation (FMD) was used to evaluate endothelial function in 109 healthy subjects. There was no correlation between serum TRAIL levels and FMD $(\rho=-0.128, p=0.148)$. 


\section{Conclusions}

Recent emerging evidence has suggested the definite involvement of TRAIL in cardiovascular diseases. Taken together, these results have shown that lower serum TRAIL levels appear to be associated with worse prognosis in patients with CAD and HF. One of most important question is why TRAIL levels are lower in such conditions. It is necessary to investigate how TRAIL is produced and cleared in humans in future studies. In addition, to confirm the direct effects of TRAIL on cardiovascular diseases, administration of recombinant TRAIL may be a powerful approach. In fact, recombinant TRAIL induces apoptosis in a wide variety of tumor cells and prevents tumor progression and metastasis in the field of cancer therapy (Johnstone et al., 2008; Wang, 2008; Wu et al., 2004). Further studies are needed to address these problems.

\section{Acknowledgment}

This review was supported in part by a Grant-in-Aid for scientific research (No. 20591068) from the Japan Society for the Promotion of Science (to ME and KM). The authors thank Dr. Naoya Kawano, Dr. Hidenori Koyama, Ms. Yuko Kikukawa, Ms. Hisako Fujii, Dr. Sanae Fukuda, Mr. Hidekichi Tokai, Dr. Yoshinobu Hirayama, and Dr. Yasuyoshi Watanabe for their help with the study to investigate the association between TRAIL levels and FMD in healthy subjects (Fig. 3).

\section{References}

Abedin, M, et al. (2007). Relation of osteoprotegerin to coronary calcium and aortic plaque (from the Dallas Heart Study). Am J Cardiol, Vol. 99, No. 4, (February 2007) pp. 513518, ISSN 0002-9149

Afford, SC, Adams, DH. (2005). Following the TRAIL from hepatitis C virus and alcohol to fatty liver. Gut, Vol. 54, No. 11, (October 2005) pp. 1518-1520, ISSN 0017-5749

Alladina, SJ, et al. (2005). TRAIL-induced apoptosis in human vascular endothelium is regulated by phosphatidylinositol 3-kinase/Akt through the short form of cellular FLIP and Bcl-2. J Vasc Res, Vol. 42, No. 4, (June 2005) pp. 337-47, ISSN 1018-1172

Benito-Martin, A, et al. (2009). Transcriptomics illustrate a deadly TRAIL to diabetic nephropathy. Nefrologia, Vol. 29, No. 1, (February 2009) pp. 13-19, ISSN 0211-6995

Chan, J, et al. (2010). TRAIL promotes VSMC proliferation and neointima formation in a FGF-2-, Sp1 phosphorylation-, and NFkappaB-dependent manner. Circ Res, Vol. 106, No. 6, (February 2010) pp. 1061-71, ISSN 1524-4571

Clarke, MC, et al. (2006). Apoptosis of vascular smooth muscle cells induces features of plaque vulnerability in atherosclerosis. Nat Med, Vol. 12, No. 9, (August 2006) pp. 1075-1080, ISSN 1078-8956

Corallini, F, et al. (2006). TRAIL, caspases and maturation of normal and leukemic myeloid precursors. Leuk Lymphoma, Vol. 47, No. 8, (September 2006) pp. 1459-1468, ISSN 1042-8194

Corallini, F, et al. (2007). Insulin down-regulates TRAIL expression in vascular smooth muscle cells both in vivo and in vitro. J Cell Physiol, Vol. 212, No. 1, (March 2007) pp. 89-95, ISSN 0021-9541 
Corallini, F, et al. (2008). TRAIL and osteoprotegerin: a role in endothelial physiopathology? Front Biosci, Vol. 13, No. (November 2007) pp. 135-147, ISSN 1093-4715

Corretti, MC, et al. (2002). Guidelines for the ultrasound assessment of endothelialdependent flow-mediated vasodilation of the brachial artery: a report of the International Brachial Artery Reactivity Task Force. J Am Coll Cardiol, Vol. 39, No. 2, (January 2002) pp. 257-265, ISSN 0735-1097

Di Pietro, R, et al. (2006). Tumor necrosis factor-related apoptosis-inducing ligand (TRAIL) regulates endothelial nitric oxide synthase (eNOS) activity and its localization within the human vein endothelial cells (HUVEC) in culture. J Cell Biochem, Vol. 97, No. 4, (October 2005) pp. 782-794, ISSN 0730-2312

Finnberg, N, El-Deiry, WS. (2008). TRAIL death receptors as tumor suppressors and drug targets. Cell Cycle, Vol. 7, No. 11, (May 2008) pp. 1525-1528, ISSN 1551-4005

Goke, R, et al. (2000). Regulation of TRAIL-induced apoptosis by transcription factors. Cell Immunol, Vol. 201, No. 2, (June 2000) pp. 77-82, ISSN 0008-8749

Hao, C, et al. (2004). Modulation of TRAIL signaling complex. Vitam Horm, Vol. 67, No. (April 2004) pp. 81-99, ISSN 0083-6729

Hymowitz, SG, et al. (1999). Triggering cell death: the crystal structure of Apo2L/TRAIL in a complex with death receptor 5. Mol Cell, Vol. 4, No. 4, (November 1999) pp. 563571, ISSN 1097-2765

Janssen, EM, et al. (2005). CD4+ T-cell help controls CD8+ T-cell memory via TRAILmediated activation-induced cell death. Nature, Vol. 434, No. 7029, (March 2005) pp. 88-93, ISSN 1476-4687

Jin, Z, et al. (2002). Enhanced sensitivity of G1 arrested human cancer cells suggests a novel therapeutic strategy using a combination of simvastatin and TRAIL. Cell Cycle, Vol. 1, No. 1, (November 2002) pp. 82-89, ISSN 1538-4101

Johnstone, RW, et al. (2008). The TRAIL apoptotic pathway in cancer onset, progression and therapy. Nat Rev Cancer, Vol. 8, No. 10, (September 2008) pp. 782-798, ISSN 14741768

Jono, S, et al. (2002). Serum osteoprotegerin levels are associated with the presence and severity of coronary artery disease. Circulation, Vol. 106, No. 10, (September 2002) pp. 1192-1194, ISSN 1524-4539

Kaplan, MJ, et al. (2000). TRAIL (Apo2 ligand) and TWEAK (Apo3 ligand) mediate CD4+ T cell killing of antigen-presenting macrophages. J Immunol, Vol. 164, No. 6, (March 2000) pp. 2897-2904, ISSN 0022-1767

Kavurma, MM, Bennett, MR. (2008). Expression, regulation and function of trail in atherosclerosis. Biochem Pharmacol, Vol. 75, No. 7, (December 2007) pp. 1441-1450, ISSN 1873-2968

Kavurma, MM, et al. (2008). TRAIL stimulates proliferation of vascular smooth muscle cells via activation of NF-kappaB and induction of insulin-like growth factor-1 receptor. J Biol Chem, Vol. 283, No. 12, (January 2008) pp. 7754-7762, ISSN 0021-9258

Kawano, N, et al. (2011). Association of serum TRAIL levels with atherosclerosis in patients with type 2 diabetes mellitus. Diabetes Res Clin Pract, Vol. 91, No. 3, (January 2011) pp. 316-320, ISSN 1872-8227

Kiechl, S, et al. (2004). Osteoprotegerin is a risk factor for progressive atherosclerosis and cardiovascular disease. Circulation, Vol. 109, No. 18, (May 2004) pp. 2175-2180, ISSN 1524-4539 
$\mathrm{Li}$, JH, et al. (2003). TRAIL induces apoptosis and inflammatory gene expression in human endothelial cells. J Immunol, Vol. 171, No. 3, (July 2003) pp. 1526-1533, ISSN 00221767

Libby, P. (2002). Inflammation in atherosclerosis. Nature, Vol. 420, No. 6917, (December 2002) pp. 868-874, ISSN 0028-0836

Martin-Ventura, JL, et al. (2007). Trail and vascular injury. Front Biosci, Vol. 12, No. (May 2007) pp. 3656-3667, ISSN 1093-4715

Michowitz, Y, et al. (2005). The involvement of tumor necrosis factor-related apoptosisinducing ligand (TRAIL) in atherosclerosis. J Am Coll Cardiol, Vol. 45, No. 7, (April 2005) pp. 1018-1024, ISSN 0735-1097

Mori, K, et al. (2010). Association of serum TRAIL level with coronary artery disease. Thromb Res, Vol. 125, No. 4, (December 2009) pp. 322-325, ISSN 1879-2472

Niessner, A, et al. (2009). Prognostic value of apoptosis markers in advanced heart failure patients. Eur Heart J, Vol. 30, No. 7, (February 2009) pp. 789-796, ISSN 1522-9645

O'Brien, LA, et al. (2007). Activated protein C decreases tumor necrosis factor related apoptosis-inducing ligand by an EPCR- independent mechanism involving Egr1/Erk-1/2 activation. Arterioscler Thromb Vasc Biol, Vol. 27, No. 12, (October 2007) pp. 2634-2641, ISSN 1524-4636

Omland, T, et al. (2008). Circulating osteoprotegerin levels and long-term prognosis in patients with acute coronary syndromes. J Am Coll Cardiol, Vol. 51, No. 6, (February 2008) pp. 627-633, ISSN 1558-3597

Patel, S, Celermajer, DS. (2006). Assessment of vascular disease using arterial flow mediated dilatation. Pharmacol Rep, Vol. 58 Suppl, No. (March 2007) pp. 3-7, ISSN 1734-1140

Pitti, RM, et al. (1996). Induction of apoptosis by Apo-2 ligand, a new member of the tumor necrosis factor cytokine family. J Biol Chem, Vol. 271, No. 22, (May 1996) pp. 1268712690, ISSN 0021-9258

Rhee, EJ, et al. (2005). Relationship of serum osteoprotegerin levels with coronary artery disease severity, left ventricular hypertrophy and C-reactive protein. Clin Sci (Lond), Vol. 108, No. 3, (December 2004) pp. 237-243, ISSN 0143-5221

Ross, R. (1993). The pathogenesis of atherosclerosis: a perspective for the 1990s. Nature, Vol. 362, No. 6423, (April 1993) pp. 801-809, ISSN 0028-0836

Sandoo, A, et al. (2010). The endothelium and its role in regulating vascular tone. Open Cardiovasc Med J, Vol. 4, No. (February 2011) pp. 302-12, ISSN 1874-1924 (Electronic)

Sato, K, et al. (2006). TRAIL-expressing T cells induce apoptosis of vascular smooth muscle cells in the atherosclerotic plaque. J Exp Med, Vol. 203, No. 1, (January 2006) pp. 239-250, ISSN 0022-1007

Sato, K, et al. (2010). Statins reduce endothelial cell apoptosis via inhibition of TRAIL expression on activated CD4 T cells in acute coronary syndrome. Atherosclerosis, Vol. 213, No. 1, (May 2010) pp. 33-39, ISSN 1879-1484

Schoppet, M, et al. (2003). Increased osteoprotegerin serum levels in men with coronary artery disease. J Clin Endocrinol Metab, Vol. 88, No. 3, (March 2003) pp. 1024-1028, ISSN 0021-972X

Schoppet, M, et al. (2004). Localization of osteoprotegerin, tumor necrosis factor-related apoptosis-inducing ligand, and receptor activator of nuclear factor-kappaB ligand in Monckeberg's sclerosis and atherosclerosis. J Clin Endocrinol Metab, Vol. 89, No. 8, (August 2004) pp. 4104-4112, ISSN 0021-972X 
Secchiero, P, et al. (2003). TRAIL promotes the survival and proliferation of primary human vascular endothelial cells by activating the Akt and ERK pathways. Circulation, Vol. 107, No. 17, (April 2003) pp. 2250-2256, ISSN 1524-4539

Secchiero, P, et al. (2004). TRAIL promotes the survival, migration and proliferation of vascular smooth muscle cells. Cell Mol Life Sci, Vol. 61, No. 15, (August 2004) pp. 1965-1974, ISSN 1420-682X

Secchiero, P, et al. (2005). TRAIL counteracts the proadhesive activity of inflammatory cytokines in endothelial cells by down-modulating CCL8 and CXCL10 chemokine expression and release. Blood, Vol. 105, No. 9, (January 2005) pp. 3413-3419, ISSN 0006-4971

Secchiero, P, et al. (2006). Systemic tumor necrosis factor-related apoptosis-inducing ligand delivery shows antiatherosclerotic activity in apolipoprotein E-null diabetic mice. Circulation, Vol. 114, No. 14, (September 2006) pp. 1522-1530, ISSN 1524-4539

Secchiero, P, Zauli, G. (2008). The puzzling role of TRAIL in endothelial cell biology. Arterioscler Thromb Vasc Biol, Vol. 28, No. 2, (January 2008) pp. e4; author reply e5-6, ISSN 1524-4636

Secchiero, P, et al. (2009). Potential prognostic significance of decreased serum levels of TRAIL after acute myocardial infarction. PLoS One, Vol. 4, No. 2, (February 2009) pp. e4442, ISSN 1932-6203

Secchiero, P, et al. (2010). An imbalanced OPG/TRAIL ratio is associated to severe acute myocardial infarction. Atherosclerosis, Vol. 210, No. 1, (December 2009) pp. 274-277, ISSN 1879-1484

Secchiero, P, et al. (2010). Metalloproteinase 2 cleaves in vitro recombinant TRAIL: potential implications for the decreased serum levels of TRAIL after acute myocardial infarction. Atherosclerosis, Vol. 211, No. 1, (March 2010) pp. 333-336, ISSN 1879-1484

Ter Avest, E, et al. (2007). What is the role of non-invasive measurements of atherosclerosis in individual cardiovascular risk prediction? Clin Sci (Lond), Vol. 112, No. 10, (April 2007) pp. 507-516, ISSN 1470-8736

Testa, U. (2010). TRAIL/TRAIL-R in hematologic malignancies. J Cell Biochem, Vol. 110, No. 1, (March 2010) pp. 21-34, ISSN 1097-4644

Tomiyama, H, et al. (2008). The relationships of cardiovascular disease risk factors to flowmediated dilatation in Japanese subjects free of cardiovascular disease. Hypertens Res, Vol. 31, No. 11, (December 2008) pp. 2019-2025, ISSN 0916-9636

Tomiyama, H, Yamashina, A. (2010). Non-invasive vascular function tests: their pathophysiological background and clinical application. Circ J, Vol. 74, No. 1, (November 2009) pp. 24-33, ISSN 1347-4820

Vaccarezza, M, et al. (2007). A role of the TRAIL-TRAIL receptor system in the pathogenesis of diabetes. Acta Biomed, Vol. 78 Suppl 1, No. (May 2007) pp. 262-267, ISSN 03924203

Venuraju, SM, et al. (2010). Osteoprotegerin as a predictor of coronary artery disease and cardiovascular mortality and morbidity. J Am Coll Cardiol, Vol. 55, No. 19, (May 2010) pp. 2049-2061, ISSN 1558-3597

Volpato, S, et al. (2011). Association of tumor necrosis factor-related apoptosis-inducing ligand with total and cardiovascular mortality in older adults. Atherosclerosis, Vol. 215, No. 2. (December 2010) pp. 452-458, ISSN 1879-1484 
Wang, S. (2008). The promise of cancer therapeutics targeting the TNF-related apoptosisinducing ligand and TRAIL receptor pathway. Oncogene, Vol. 27, No. 48, (October 2008) pp. 6207-6215, ISSN 1476-5594

Watt, V, et al. (2011). TRAIL attenuates the development of atherosclerosis in apolipoprotein E deficient mice. Atherosclerosis, Vol. No. (February 2011) pp. ISSN 1879-1484

Weyand, CM, et al. (2008). T cells in arteritis and atherosclerosis. Curr Opin Lipidol, Vol. 19, No. 5, (October 2008) pp. 469-477, ISSN 0957-9672

Wiley, SR, et al. (1995). Identification and characterization of a new member of the TNF family that induces apoptosis. Immunity, Vol. 3, No. 6, (December 1995) pp. 673682, ISSN 1074-7613

$\mathrm{Wu}, \mathrm{XX}$, et al. (2004). TRAIL and chemotherapeutic drugs in cancer therapy. Vitam Horm, Vol. 67, No. (April 2004) pp. 365-383, ISSN 0083-6729

Zauli, G, et al. (2003). Tumor necrosis factor-related apoptosis-inducing ligand (TRAIL) sequentially upregulates nitric oxide and prostanoid production in primary human endothelial cells. Circ Res, Vol. 92, No. 7, (March 2003) pp. 732-740, ISSN 1524-4571 


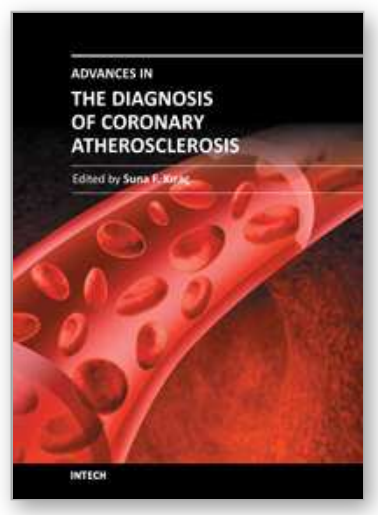

\author{
Advances in the Diagnosis of Coronary Atherosclerosis \\ Edited by Prof. Suna Kirac
}

ISBN 978-953-307-286-9

Hard cover, 378 pages

Publisher InTech

Published online 09, November, 2011

Published in print edition November, 2011

Coronary artery disease $(C A D)$ and its consequences are most important morbidity and mortality reasons in the developed and developing countries. To prevent hard end-points, early definitive diagnosis and optimum therapy play significant role. Novel advanced diagnostic tests which are biomarkers of inflammation, cell adhesion, cell activation and imaging techniques provide to get the best result in the detection and characterization of calcified or uncalcified atherosclerotic plaques. In spite of last developments in the imaging methods, coronary catheterization is still frequently performed. Following the first cardiac catheterization performed in 1844, date by date historical developments and the mechanics of cardiac catheterization techniques, risks associated with coronary angiography, and also, preventions and treatments of possible complications have been presented in this book. Other important issue is radiation exposure of patients and staff during coronary angiography and scintigraphy. Radiation dose reduction techniques, general radiation protection principles have been discussed in related chapters.

\title{
How to reference
}

In order to correctly reference this scholarly work, feel free to copy and paste the following:

Katsuhito Mori, Masanori Emoto and Masaaki Inaba (2011). Multifunctional Role of TRAIL in Atherosclerosis and Cardiovascular Disease, Advances in the Diagnosis of Coronary Atherosclerosis, Prof. Suna Kirac (Ed.), ISBN: 978-953-307-286-9, InTech, Available from: http://www.intechopen.com/books/advances-in-thediagnosis-of-coronary-atherosclerosis/multifunctional-role-of-trail-in-atherosclerosis-and-cardiovasculardisease

\section{INTECH}

open science | open minds

\section{InTech Europe}

University Campus STeP Ri

Slavka Krautzeka 83/A

51000 Rijeka, Croatia

Phone: +385 (51) 770447

Fax: +385 (51) 686166

www.intechopen.com

\section{InTech China}

Unit 405, Office Block, Hotel Equatorial Shanghai

No.65, Yan An Road (West), Shanghai, 200040, China 中国上海市延安西路65号上海国际贵都大饭店办公楼 405 单元

Phone: +86-21-62489820

Fax: +86-21-62489821 
(C) 2011 The Author(s). Licensee IntechOpen. This is an open access article distributed under the terms of the Creative Commons Attribution 3.0 License, which permits unrestricted use, distribution, and reproduction in any medium, provided the original work is properly cited. 